\title{
A Novel Rational Harmonic Balance Approach for Periodic Solutions of Conservative Nonlinear Oscillators
}

\author{
A. Beléndez, E. Gimeno, M. L. Álvarez, S. Gallego, M. Ortuño and D. I. Méndez \\ Departamento de Física, Ingeniería de Sistemas y Teoría de la Señal. \\ Universidad de Alicante. Apartado 99. E-03080 Alicante. SPAIN \\ E-mail: a.belendez@ua.es,Fax:+34-96-5909750
}

\begin{abstract}
An analytical approximate procedure for a class of conservative single degree-of-freedom nonlinear oscillators with odd non-linearity is proposed. This technique is based on the generalized harmonic balance method in which analytical approximate solutions have rational forms. Unlike the classical harmonic balance techniques, in this new procedure the approximate solution and the restoring force are expanded in Fourier series prior to substituting them in the nonlinear differential equation. This approach gives us not only a truly periodic solution but also the frequency of the motion as a function of the amplitude of oscillation. Four nonlinear oscillators are presented to illustrate the usefulness and effectiveness of the proposed technique. The most significant features of this method are its simplicity and its excellent accuracy for the whole range of oscillation amplitude values and the results reveal that this technique is very effective and convenient for solving a class of conservative nonlinear oscillatory systems.
\end{abstract}

Keywords: Nonlinear oscillator; Analytical approximations; Rational harmonic balance method; Fourier series.

\section{Introduction}

Physical and mechanical oscillatory systems are often governed by nonlinear differential equations. It is very difficult to solve nonlinear problems and, in general, it is often more difficult to get an analytic approximation than a numerical one for a given nonlinear problem. In particular the study of nonlinear oscillators is of great interest to many researchers. A large variety of approximate methods is commonly used for solving nonlinear oscillatory systems. The most commons and most widely studied methods of all approximation methods for nonlinear differential equations are perturbation methods [1,2]. Some of other techniques include variational and variational iteration methods [3-15], expfunction [16-19], homotopy perturbation [20$35]$, equivalent linearization [36,37], standard and modified Lindstedt-Poincaré [38-44], artificial parameter [45,46], parameter expanding [47,50], harmonic balance methods [1,51-56], etc. Surveys of the literature with numerous references and useful bibliography and a review of these methods can be found in detail in [44] and [57].

The method of harmonic balance is a wellestablished procedure for determining analytical approximations to the solutions of differential equations, the time domain response of which can be expressed as a Fourier series. In the usual harmonic balance methods, the solution of a nonlinear system is assumed to be of the form of a truncated Fourier series [1]. Being different from the other non-linear analytical methods, such as perturbation techniques, the harmonic balance method does not depend on small parameters, such that it can find wide application in nonlinear problems without linearization or small perturbations. Various modifications of the harmonic balance method 
have been made and one of them is the rational representation proposed by Mickens [59-61].

In this paper a new procedure to apply the generalized, rational harmonic balance method is proposed for constructing approximate analytical solutions to conservative nonlinear oscillations in which the nonlinear restoring force is an odd function of the displacement. In this method the approximate solution obtained approximates all of the harmonics in the exact solution [61], whereas the usual harmonic balance techniques provide an approximation to only the lowest harmonic components. Unlike the classical harmonic balance techniques, in this new procedure the approximate solution and the restoring force are expanded in Fourier series prior to substituting them in the nonlinear differential equation. The most interesting features of the proposed method are its simplicity and its excellent accuracy in a wide range of values of oscillation amplitude. We present four examples to illustrate the applicability and the effectiveness of the proposed approximate analytical solutions.

\section{Solution procedure}

Consider the following single degree-offreedom conservative nonlinear oscillator

$$
\begin{gathered}
\frac{\mathrm{d}^{2} x}{\mathrm{~d} t^{2}}+f(x)=0 \\
x(0)=A, \quad \frac{\mathrm{d} x(0)}{\mathrm{d} t}=0
\end{gathered}
$$

where the nonlinear function $f(x)$ is odd, i.e., $f(-x)=-f(x)$, and satisfies $x f(x)>0$ for $x \in[-A, A], x \neq 0$. The equilibrium position is $x$ $=0$, the system oscillates between symmetric bounds $[+A,-A]$, and its frequency and the corresponding periodic solution are dependent on the amplitude $A$.

A new independent variable $\tau=\omega t$ is introduced, and then Eqs. (1) and (2) can be rewritten as follows

$$
\begin{gathered}
\omega^{2} \frac{\mathrm{d}^{2} x}{\mathrm{~d} \tau^{2}}+f(x)=0 \\
x(0)=A, \quad \frac{\mathrm{d} x}{\mathrm{~d} \tau}(0)=0
\end{gathered}
$$

The new independent variable is chosen in such a way that the solution of Eq. (3) is a periodic function of $\tau$ of period $2 \pi$. The corresponding frequency of the nonlinear oscillator is $\omega$ and both periodic solution $x(\tau)$ and frequency $\omega$ depend on the initial amplitude $A$.

Following the lowest order harmonic balance approximation, we set

$$
x_{1}(\tau)=A \cos \tau
$$

which satisfies the initial conditions in Eq. (4). Substituting Eq. (5) into Eq. (3) and setting the coefficient of resulting $\cos \tau$ to zero yield the first approximation to the frequency in terms of the amplitude $A$

where

$$
\omega_{1}(A)=\sqrt{\frac{c_{1}}{A}}
$$

$$
c_{1}=\frac{4}{\pi} \int_{0}^{\pi / 2} f\left(x_{1}(\tau)\right) \cos \tau \mathrm{d} \tau
$$

is the first coefficient of the Fourier series expansion of function $f\left(x_{1}(\tau)\right)$

$$
f\left(x_{1}(\tau)\right)=\sum_{n=0}^{\infty} c_{2 n+1} \cos [(2 n+1) \tau]
$$

where only odd multiples of are presented because the nonlinear function $f(x)$ is odd.

In order to determine an improved approximation we use a generalized, rational form given by the following expression $[61,62]$

$$
x_{2}(\tau)=\frac{A_{1} \cos \tau}{1+B_{2} \cos 2 \tau}
$$

In this equation $A_{1}, B_{2}$ and $\omega$ are to be determined as functions of the initial conditions expressed in Eq. (4) and $\left|B_{2}\right|<1$. From Eq. (4) we obtain $A_{1}=\left(1+B_{2}\right) A$ and Eq. (9) can be rewritten as follows

$$
x_{2}(\tau)=\frac{\left(1+B_{2}\right) A \cos \tau}{1+B_{2} \cos 2 \tau}
$$

The Fourier representation of Eq. (17) is

$$
x_{2}(\tau)=\sum_{n=0}^{\infty} a_{2 n+1} \cos [(2 n+1) \tau]
$$


and the following result is obtained for the Fourier coefficients $a_{2 n+1}$ [62]

$$
\begin{aligned}
& a_{2 n+1}= \\
& =(-1)^{n} 2^{n+1} A \sqrt{\frac{1+B_{2}}{1-B_{2}}}\left(\frac{B_{2}}{1-B_{2}}\right)^{n}\left(\frac{\sqrt{1-B_{2}}}{\sqrt{1-B_{2}}+\sqrt{1+B_{2}}}\right)^{2 n+1}
\end{aligned}
$$

Based on the odd function assumption, $f(-x)=-f(-x), f\left(x_{2}(\tau)\right)$ can be expanded in a Fourier series as

$$
f\left(x_{2}(\tau)\right)=\sum_{n=0}^{\infty} b_{2 n+1} \cos [(2 n+1) \tau]
$$
yields

Substituting Eqs. (11) and (13) into Eq. (3)

$$
\begin{aligned}
-\omega^{2} \sum_{n=0}^{\infty}(2 n & +1)^{2} a_{2 n+1} \cos [(2 n+1) \tau] \\
+\sum_{n=0}^{\infty} b_{2 n+1} \cos [(2 n+1) \tau] & =0
\end{aligned}
$$

and setting the coefficients of $\cos \tau$ and $\cos 3 \tau$ to zeros, respectively, yields

$$
\begin{gathered}
-\omega^{2} a_{1}\left(A, B_{2}\right)+b_{1}\left(A, B_{2}\right)=0 \\
-9 \omega^{2} a_{3}\left(A, B_{2}\right)+b_{3}\left(A, B_{2}\right)=0
\end{gathered}
$$

From Eq. (12), we obtain

$$
\begin{gathered}
a_{1}\left(A, B_{2}\right)=\frac{2 A \sqrt{1+B_{2}}}{\sqrt{1-B_{2}}+\sqrt{1+B_{2}}} \\
a_{3}\left(A, B_{2}\right)=-\frac{4 A B_{2} \sqrt{1+B_{2}}}{\left(\sqrt{1-B_{2}}+\sqrt{1+B_{2}}\right)^{3}}
\end{gathered}
$$

Solving Eqs. (15) and (16) we can obtain $B_{2}$ and the second order approximate frequency $\omega$ as a function of $A$. It should be clear how the procedure works for constructing the secondorder analytical approximate solution. As you can see, the main problem is to obtain the
Fourier series expansion for $f\left(x_{2}(\tau)\right)$. To do this, we re-write Eq. (10) as follows

$$
\begin{gathered}
x_{2}(\tau)=\frac{\left(1+B_{2}\right) A \cos \tau}{1+2 B_{2} \cos ^{2} \tau-B_{2}} \\
=\frac{\left(1+B_{2}\right) A \cos \tau}{\left(1-B_{2}\right)\left(1+\frac{2 B_{2}}{1-B_{2}} \cos ^{2} \tau\right)} \\
=\frac{\left(1+B_{2}\right) A}{\sqrt{2 B_{2}\left(1-B_{2}\right)}} \frac{u}{1+u^{2}}
\end{gathered}
$$

where

$$
u=\sqrt{\frac{2 B_{2}}{1-B_{2}}} \cos \tau
$$

As $\left|B_{2}\right|<<1$ we obtain $|u|<<1$. We will show in the following examples that this new technique provides excellent analytical approximations to frequency and corresponding periodic solutions for a class of conservative nonlinear oscillators.

\section{Illustrative examples}

In this section, we present four examples to illustrate the usefulness and effectiveness of the proposed technique.

Example 1. Cubic truly nonlinear oscillator.

This oscillator is governed by the following differential equation

$$
\frac{\mathrm{d}^{2} x}{\mathrm{~d} t^{2}}+x^{3}=0, \quad f(x)=x^{3}
$$

with initial conditions given in Eq. (2).

From Eq. (19) we can be write

$$
f\left(x_{2}(\tau)\right)=x_{2}^{3}(\tau)=\frac{A^{3}\left(1+B_{2}\right)^{3}}{\sqrt{8 B_{2}^{3}\left(1-B_{2}\right)^{3}}} \frac{u^{3}}{\left(1+u^{2}\right)^{3}}
$$

Now applying the Taylor series expansion, it follows that

$$
\frac{u^{3}}{\left(1+u^{2}\right)^{3}}=\sum_{n=0}^{\infty} \frac{(-1)^{n}(n+2) !}{2 n !} u^{2 n+3}
$$


Substituting Eqs. (20) and (23) into Eq. (22), it can be derived that

$$
\begin{gathered}
f\left(x_{2}(\tau)\right)=\sum_{n=0}^{\infty} \frac{(-1)^{n}(n+2) ! 2^{n-1} B_{2}^{n}\left(1+B_{2}\right)^{3} A^{3}}{\left(1-B_{2}\right)^{n+3} n !} \cos ^{2 n+3} \tau \\
=\sum_{n=0}^{\infty} d_{n} \cos ^{2 n+3} \tau
\end{gathered}
$$

The formula that allows us to obtain the odd power of the cosine is

$$
\begin{aligned}
\cos ^{2 n+3} \tau & =\frac{1}{2^{2 n+2}} \sum_{j=0}^{n+1}\left(\begin{array}{c}
2 n+3 \\
n+1-j
\end{array}\right) \cos [(2 j+1) \tau] \\
& =\sum_{j=0}^{n+1} h_{j}^{(n)} \cos [(2 j+1) \tau]
\end{aligned}
$$

Substituting Eq. (26) into Eq. (24) gives

$$
\begin{gathered}
f\left(x_{2}(\tau)\right)=\sum_{n=0}^{\infty} \sum_{j=0}^{n+1} d_{n} h_{j}^{(n)} \cos [(2 j+1) \tau]= \\
=\sum_{n=0}^{\infty} d_{n} h_{0}^{(n)} \cos \tau+\sum_{n=0}^{\infty} d_{n} h_{1}^{(n)} \cos 3 \tau+ \\
+\sum_{n=1}^{\infty} d_{n} h_{2}^{(n)} \cos 5 \tau+\sum_{n=2}^{\infty} d_{n} h_{3}^{(n)} \cos 7 \tau+\ldots
\end{gathered}
$$

From Eqs. (13), (24), (25) and (26) we obtain $b_{1}$ and $b_{3}$ as follows

$$
\begin{gathered}
b_{1}=\frac{3}{4} A^{3} \Delta \\
b_{3}=\frac{A^{3}}{4 B_{2}^{3}}\left[4-4 \Delta+(12-8 \Delta) B_{2}\right. \\
\left.-2(-6+\Delta) B_{2}^{2}+(4+5 \Delta) B_{2}^{3}\right]
\end{gathered}
$$

where

$$
\Delta=\sqrt{\frac{1+B_{2}}{1-B_{2}}}
$$

Substituting Eqs. (17), (18), (27), (28) and (29) into Eqs. (15) and (16), and solving the resulting equations for $B_{2}$ and $\omega$, we obtain

$$
\omega_{2}(A)=A \sqrt{\frac{3}{8}\left(1+\sqrt{\frac{1+B_{2}}{1-B_{2}}}\right)}=0.847138 A
$$

As we can see, for $B_{2}=0$ the approximate frequency for the first-order harmonic balance approximation is obtained $\left(\omega_{1}=\sqrt{3 / 8} A\right)$.

Therefore, the second approximation to the periodic solution of the nonlinear oscillator is given by the following equation

$$
\frac{x_{2}(t)}{A}=\frac{0.9100048 \cos (0.847138 A t)}{1-0.0899952 \cos (1.694276 A t)}
$$

From Eqs. (11) and (12) the Fourier series expansion for the second-order approximate solution given in Eq. (32) is

$$
\begin{aligned}
& \frac{x_{2}(t)}{A}=0.954911 \cos \omega_{2} t+0.043056 \cos 3 \omega_{2} t \\
& \quad+0.00194136 \cos 5 \omega_{2} t+0.0000875340 \cos 7 \omega_{2} t+\ldots
\end{aligned}
$$

which has an infinite number of harmonics.

For this problem the exact frequency is [27]

$$
\omega_{e}(A)=\frac{\pi A}{2 K(1 / 2)}=0.847213 A
$$

where $K(m)$ is the complete elliptic integral of the first kind defined as follows

$$
K(m)=\int_{0}^{1} \frac{\mathrm{d} z}{\sqrt{\left(1-z^{2}\right)\left(1-m z^{2}\right)}}
$$

The exact solution to Eq. (21) is [27]

$$
\frac{x_{e}(t)}{A}=\operatorname{cn}(A t ; 1 / 2)
$$

where $c n$ is the Jacobi elliptic function which has the following Fourier expansion $[1,27]$

$$
c n(u ; m)=\frac{2 \pi}{\sqrt{m} K(m)} \sum_{n=0}^{\infty} \frac{q^{n+1 / 2}(m)}{1+q^{2 n+1}(m)} \cos \left[\frac{(2 n+1) \pi u}{2 K(m)}\right]
$$

where

$$
q(m)=\exp \left[-\frac{\pi K\left(m^{\prime}\right)}{K(m)}\right]
$$

and $m^{\prime}=1-m$. With these results, the Fourier expansion of Eq. (36) becomes 


$$
\begin{aligned}
& \frac{x_{e}(t)}{A}=\operatorname{cn}(A t ; 1 / 2)= \\
& =\frac{2 \pi \sqrt{2}}{K(1 / 2)} \sum_{n=0}^{\infty}\left(\frac{\exp [(n+1 / 2) \pi]}{1+\exp [(2 n+1) \pi]}\right) \cos \left[(2 n+1) \omega_{e} t\right]= \\
& =0.95501 \cos \omega_{e} t+0.043050 \cos 3 \omega_{e} t+ \\
& +0.0018605 \cos 5 \omega_{e} t+0.0000804 \cos 7 \omega_{e} t+\ldots \text { (39) }
\end{aligned}
$$

If we compare the first four terms of the Fourier series expansions of $x_{2}(t) / A$ (Eq. (33) and $x_{e}(t) / A$ (Eq. (39)) we can see that the relative errors for these terms are $0.010 \%$, $0.014 \%, 4.3 \%$ and $8.9 \%$, respectively.

The percentage error $(P E)$ of the approximate frequency $\omega_{2}$ in relation to the exact one is

$$
P E=\left|\left(\omega_{e}-\omega_{2}\right) / \omega_{e}\right| \cdot 100=0.0089 \%
$$

The $0.0089 \%$ accuracy is remarkably very good.

By applying the second approximation based on the standard method of harmonic balance method, Mickens achieved the following expression for the frequency [1]

$$
\omega_{M 2}(A)=0.8507 A \quad(P E=0.41 \%)
$$

$\mathrm{Wu}$ et al. [53] approximately solved Eq. (21) using an improved harmonic balance method (LHBM) that incorporates salient features of both Newton's method and the harmonic balance method. They achieved the following results for the second and third approximation orders

$$
\begin{gathered}
\omega_{W S L 2}(A)=\sqrt{\frac{23}{32}} A=0.847791 A(P E=0.068 \%) \\
\omega_{W S L 3}(A)=\sqrt{\frac{65856986475}{91739270448}} A=0.847273 A \\
(P E=0.0070 \%)
\end{gathered}
$$

Beléndez et al. [27] approximately solved Eq. (21) using He's homotopy perturbation method (HPM). They achieved the following results for the second and third approximation orders

$$
\omega_{B 2}(A)=\omega_{B 3}(A)=\frac{1}{4} \sqrt{6+\sqrt{30}} A=0.84695136 A
$$$$
(P E=0.031 \%)(43)
$$

The normalized periodic exact solution, $x_{e}(t) / A$, achieved using Eq. (36), and the proposed second-order approximate solution, $x_{2}(t) / A$ (Eq. (32)), are plotted in Figure 1, whereas in Figure 2 we plotted the difference $\left(x_{e}-x_{2}\right) / A$. In these figures $h$ is defined as $h=t / T_{e}$.

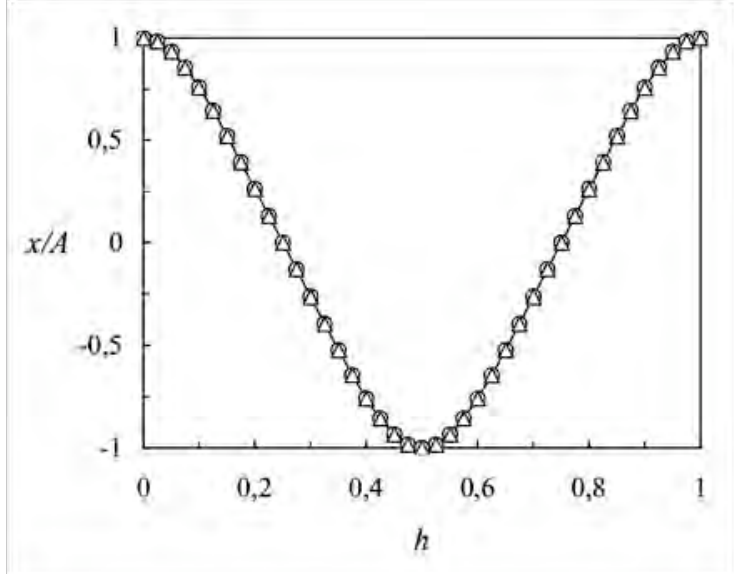

Fig. 1 Normalized approximate solution (triangles and dashed line) and exact solution (circles and continuous line) for the example 1.

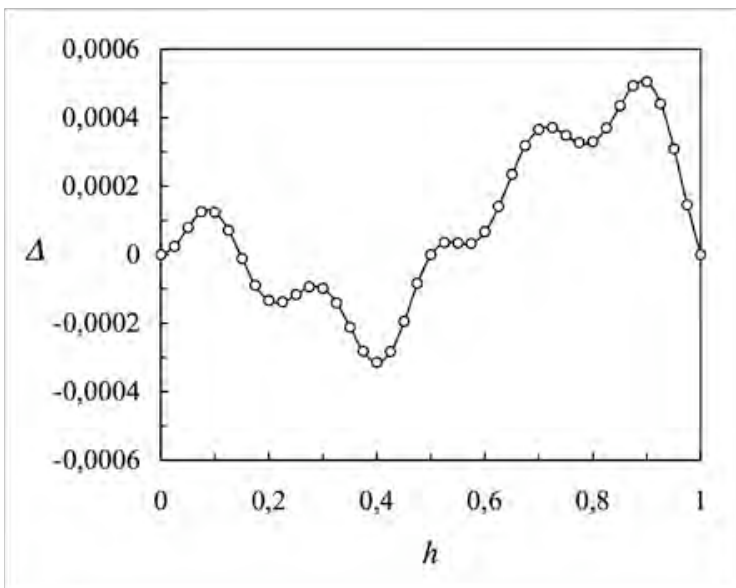

Fig. 2 Difference between normalized exact and second order approximate solutions (example 1).

Example 2. Cubic Duffing nonlinear oscillator. This oscillator is governed by the following differential equation

$$
\frac{\mathrm{d}^{2} x}{\mathrm{~d} t^{2}}+x+\varepsilon x^{3}=0, \quad f(x)=x+\varepsilon x^{3}
$$

with initial conditions given in Eq. (2). 
From Eq. (19) we can be write

$$
\begin{aligned}
& f\left(x_{2}(\tau)\right)=x_{2}(\tau)+\varepsilon x_{2}^{3}(\tau) \\
& =\frac{A\left(1+B_{2}\right)}{\sqrt{2 B_{2}\left(1-B_{2}\right)}} \frac{u}{1+u^{2}}+\varepsilon \frac{A^{3}\left(1+B_{2}\right)^{3}}{\sqrt{8 B_{2}^{3}\left(1-B_{2}\right)^{3}}} \frac{u^{3}}{\left(1+u^{2}\right)^{3}}
\end{aligned}
$$

From Eqs. (11), (27), (28) and (45) we can easily obtain

$$
\begin{aligned}
b_{1}= & a_{1}+\frac{3}{4} \varepsilon A^{3} \Delta \\
b_{3}= & a_{3}+\frac{\varepsilon A^{3}}{4 B_{2}^{3}}\left[4-4 \Delta+(12-8 \Delta) B_{2}\right. \\
& \left.-2(-6+\Delta) B_{2}^{2}+(4+5 \Delta) B_{2}^{3}\right]
\end{aligned}
$$

where $a_{1}, a_{2}$ and $\Delta$ are given by Eqs. (17), (18) and (29), respectively. Substituting Eqs. (17), (18), (46), (47) and (29) into Eqs. (15) and (16), we can obtain $\omega$ for different values of $\varepsilon$ and $A$

$$
\omega_{2}(A)=\sqrt{1+\frac{3}{8} \varepsilon A^{2}\left(1+\sqrt{\frac{1+B_{2}}{1-B_{2}}}\right)}
$$

where $B_{2}$ is the solution of Eq. (16) when $\omega$ is given by Eq. (48), i.e.

$$
\begin{gathered}
\frac{2 B_{2} \sqrt{1+B_{2}}}{\left(\sqrt{1-B_{2}}+\sqrt{1+B_{2}}\right)^{3}}\left[64+27 \varepsilon A^{2}\left(1+\sqrt{\frac{1+B_{2}}{1-B_{2}}}\right)\right]+ \\
+\frac{\varepsilon A^{2}}{B_{2}^{3}}\left[4-4 \sqrt{\frac{1+B_{2}}{1-B_{2}}}+B_{2}\left(12-8 \sqrt{\frac{1+B_{2}}{1-B_{2}}}\right)\right. \\
\left.+2 B_{2}^{2}\left(6-\sqrt{\frac{1+B_{2}}{1-B_{2}}}\right)+B_{2}^{3}\left(4+5 \sqrt{\frac{1+B_{2}}{1-B_{2}}}\right)\right]=0
\end{gathered}
$$

which allows us to obtain $B_{2}$ as a function of $\varepsilon A^{2}$. From Eqs. (49) and (29) we obtain

$$
\begin{gathered}
\lim _{\varepsilon A^{2} \rightarrow \infty} B_{2}=-0.0899952 \\
\lim _{\varepsilon A^{2} \rightarrow \infty} \Delta=0.9137125
\end{gathered}
$$

In Figure 3 we have plotted $B_{2}$ as a function of $\varepsilon A^{2}$. As we can see $B_{2}$ is only defined for $\varepsilon A^{2}>-1$. It should be noted that the maximum amplitude of the Duffing oscillator in Eq. (44) for $\varepsilon=-1$, is $A=1$ which corresponds to the heteroclinic orbit with period $+\infty$ [53].

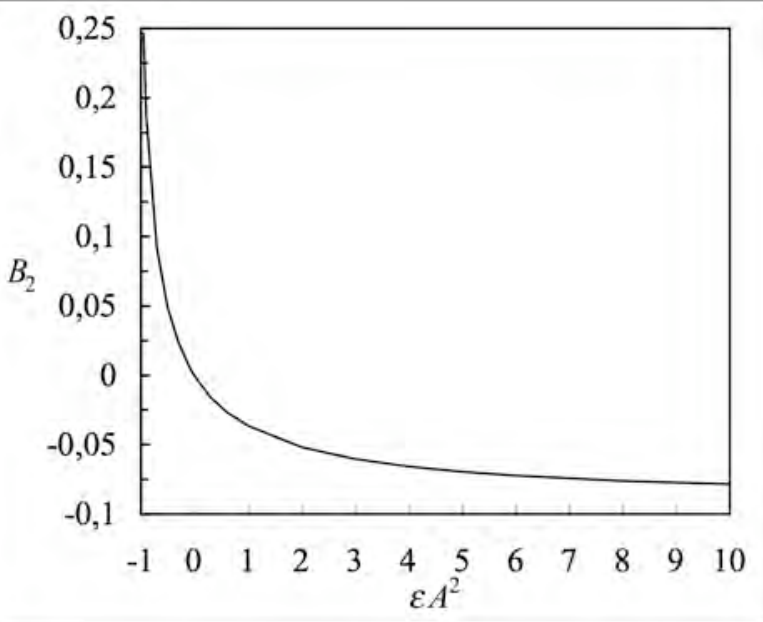

Fig. $3 B_{2}$ as a function of $\varepsilon A^{2}$ for the example 2 .

As we can see in Eq. (48), for $B_{2}=0$, the approximate frequency for the first-order harmonic balance approximation is obtained [1]

$$
\omega_{1}(A)=\sqrt{1+\frac{3}{4} \varepsilon A^{2}}
$$

The second approximation to the periodic solution of this nonlinear oscillator for the value $\varepsilon A^{2}=10$ is given by the following equation

$$
\frac{x_{2}(t)}{A}=\frac{0.9216309 \cos (2.866492 t)}{1-0.0783691 \cos (5.732984 t)}
$$

From Eqs. (11) and (12) the Fourier series expansion for the second-order approximate solution given in Eq. (52) for $\varepsilon A^{2}=10$ is

$$
\begin{aligned}
& \frac{x_{2}(t)}{A}=0.960755 \cos \omega_{2} t+0.0377047 \cos 3 \omega_{2} t+ \\
& \quad+0.00147972 \cos 5 \omega_{2} t+0.0000580714 \cos 7 \omega_{2} t+\ldots
\end{aligned}
$$

which has an infinite number of harmonics. 
For this problem the exact frequency is [1]

$$
\omega_{e}(A)=\frac{\pi \sqrt{1+\varepsilon A^{2}}}{2 K(k)}
$$

where

$$
k=\frac{\varepsilon A^{2}}{2\left(1+\varepsilon A^{2}\right)}
$$

and the exact solution to Eq. (44) is given as follows

$$
\frac{x_{e}(t)}{A}=c n\left[t\left(1+\varepsilon A^{2}\right)^{1 / 2} ; k\right]
$$

where $c n$ is the Jacobi elliptic function which has the following Fourier expansion [1]

$$
\frac{x_{e}(t)}{A}=\frac{2 \pi}{k K(k)} \sum_{n=0}^{\infty} \frac{q^{n+1 / 2}(k)}{1+q^{2 n+1}(k)} \cos \left[(2 n+1) \omega_{e} t\right]
$$

where $q$ is given by Eq. (38). The first four terms of the Fourier expansion of Eq. (57) for $\varepsilon A^{2}=10$ are

$$
\begin{aligned}
& \frac{x_{e}(t)}{A}=0.960817 \cos \omega_{e} t+0.0377014 \cos 3 \omega_{e} t+ \\
& +0.00142562 \cos 5 \omega_{e} t+0.0000539044 \cos 7 \omega_{e} t+\ldots
\end{aligned}
$$

If we compare the first four terms of the Fourier series expansions of $x_{2}(t) / A$ (Eq. (52) and $x_{e}(t) / A$ (Eq. (58)) for $\varepsilon A^{2}=10$, we can see that the relative errors for these terms are $0.0064 \%$, $0.0089 \%, 3.8 \%$ and $7.7 \%$, respectively.

From Eqs. (48), (50) and (54) we have

$$
\lim _{\varepsilon A^{2} \rightarrow \infty} \frac{\omega_{2}}{\omega_{e}}=\frac{2}{\pi} \sqrt{\frac{3}{8} 1.9137125} K(1 / 2)=0.999911
$$

Wu et al. [53] approximately solved Eq. (44) using an improved harmonic balance method (LHBM). They achieved the following results for the second approximation order
$\omega_{W S L 2}(A)=\sqrt{\frac{128+192 \varepsilon A^{2}+69 \varepsilon^{2} A^{4}}{128+96 \varepsilon A^{2}}}$

and

$\lim _{\varepsilon A^{2} \rightarrow \infty} \frac{\omega_{W S L 2}}{\omega_{e}}=\frac{1}{\pi} \sqrt{\frac{69}{24}} K(1 / 2)=1.000682$

In Figure 4 we plotted the relative errors for the approximate frequencies $\omega_{2}$ and $\omega_{W S L 2}$. This figure indicates that $\omega_{2}$ is more accurate than $\omega_{W S L 2}$ and provides excellent approximations to the exact frequency for the whole range of parameter $\varepsilon A^{2}>-1$.

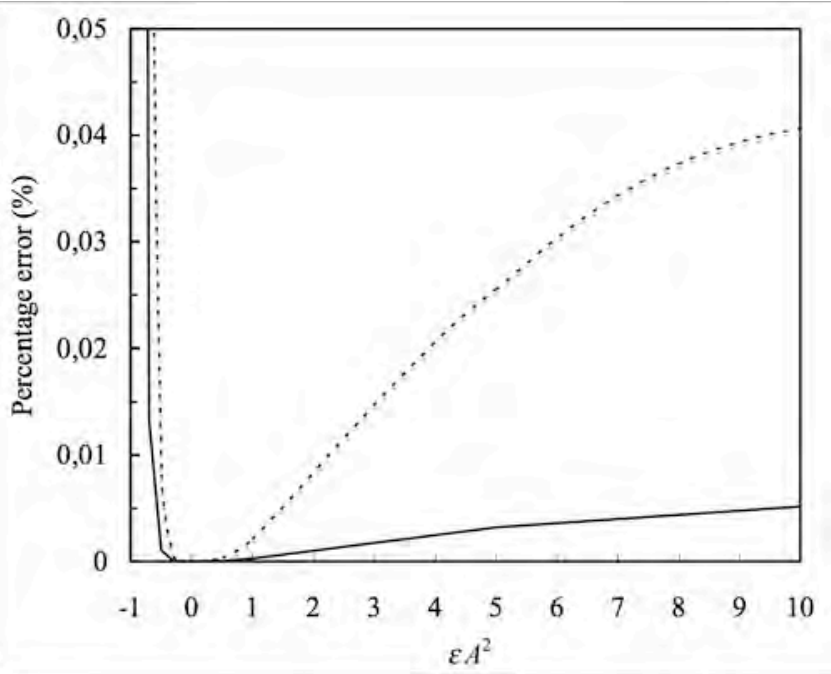

Fig. 4 Relative error for the approximate frequencies $\omega_{W S L 2}$ (dashed line) and $\omega_{2}$ (continuous line).

For $\varepsilon A^{2}=-0.9,10$ and 100 , the exact periodic solution $x_{e} / A$ obtained using Eq. (56) and the approximate periodic solution $x_{2} / A$ computed by Eq. (52), are plotted as a function of $h=t / T_{e}$ in Figures 5, 6 and 7, respectively, whereas in Figures 8,9 and 10 we plotted the difference $\left(x_{e}-x_{2}\right) / A$. These figures show that the proposed formulas (48) and (52) provide excellent approximations to exact periodic solutions for both soft and hard nonlinear oscillators. 


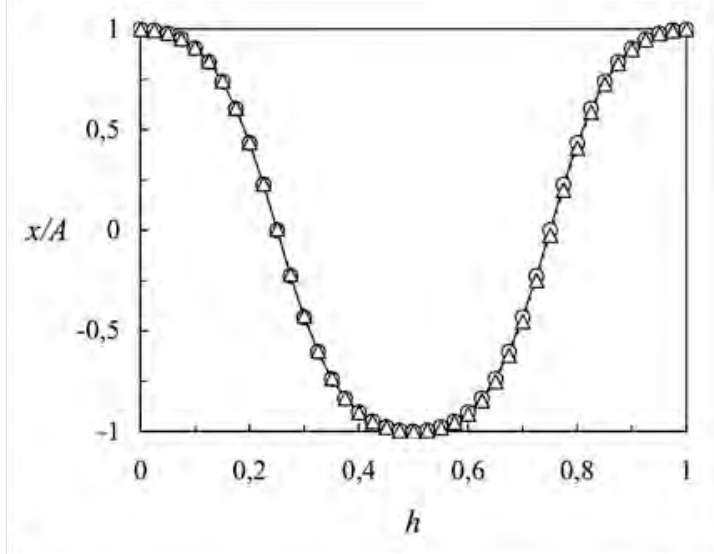

Fig. 5 Normalized approximate solution (triangles and dashed line) and exact solution (circles and continuous line) for $\varepsilon A^{2}=-0.9$.

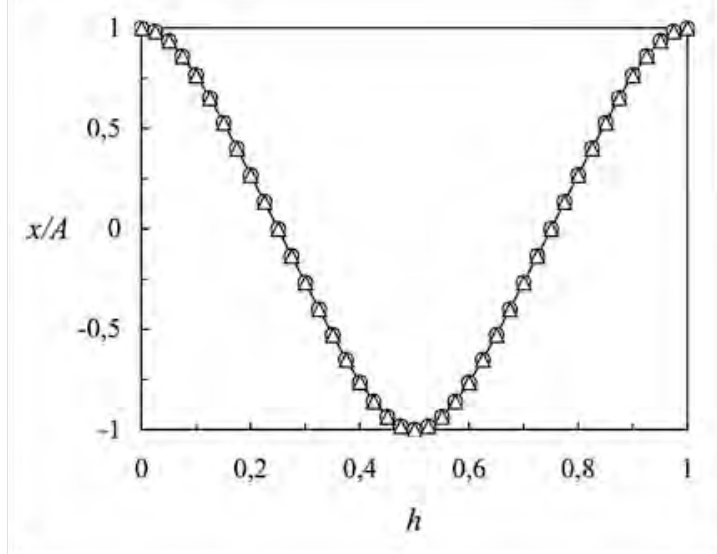

Fig. 6 Normalized approximate solution (triangles and dashed line) and exact solution (circles and continuous line) for $\varepsilon A^{2}=10$.

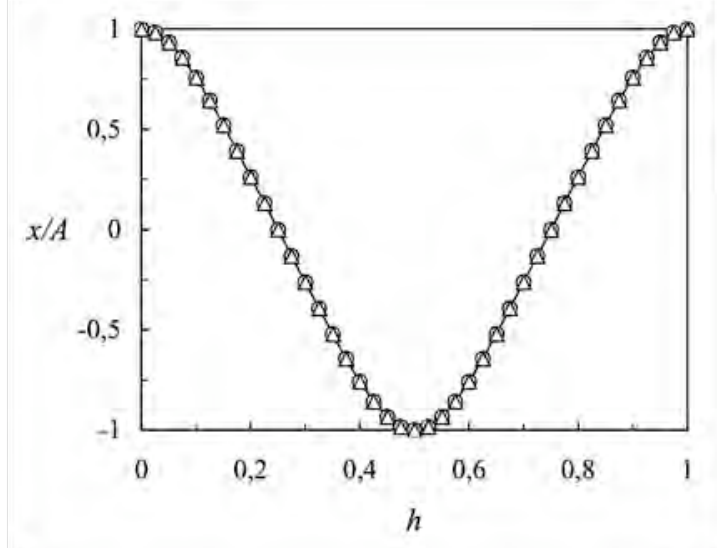

Fig. 7 Normalized approximate solution (triangles and dashed line) and exact solution (circles and continuous line) for $\varepsilon A^{2}=100$.

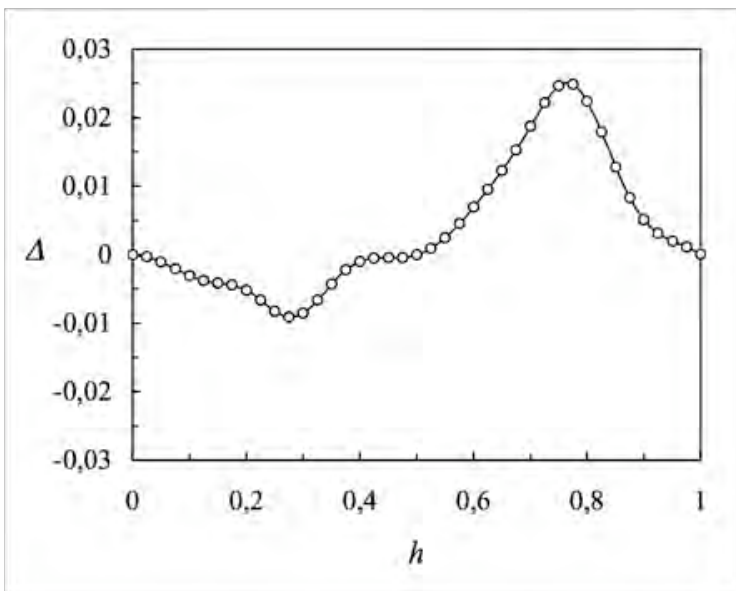

Fig. 8 Difference between normalized exact and approximate solutions for $\varepsilon A^{2}=-0.9$.

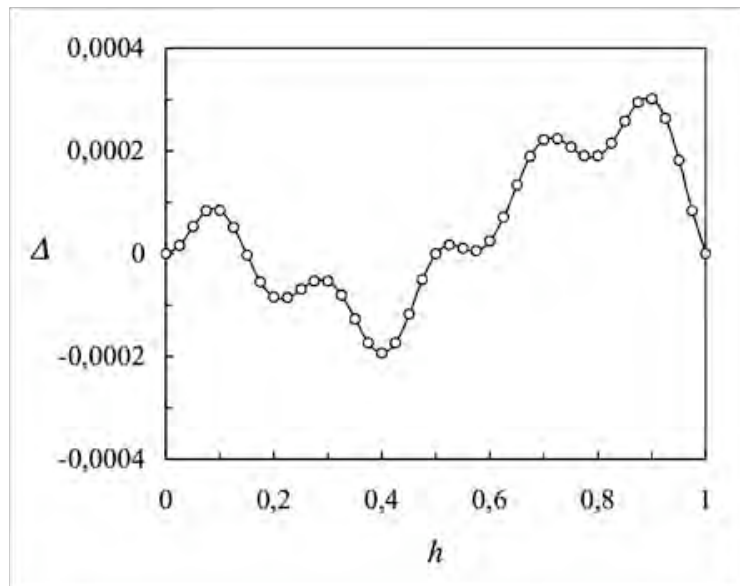

Fig. 9 Difference between normalized exact and approximate solutions for $\varepsilon A^{2}=10$.

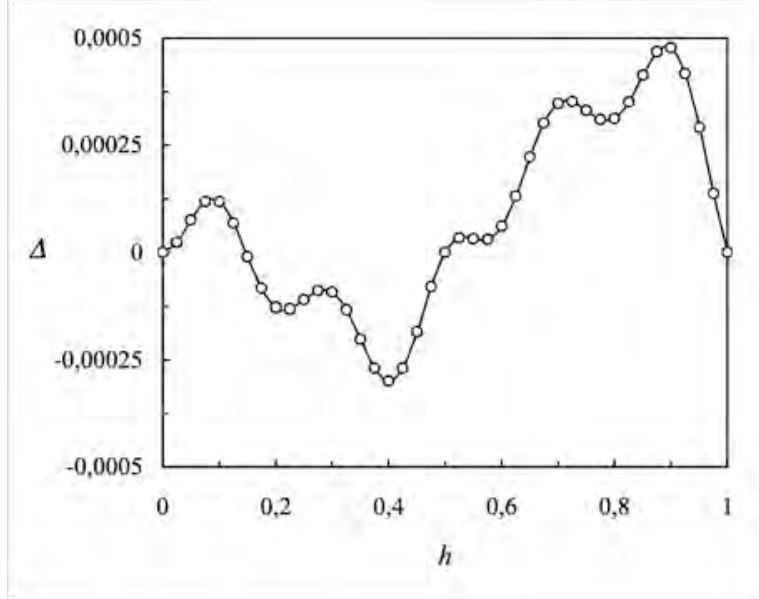

Fig. 10 Difference between normalized exact and approximate solutions for $\varepsilon A^{2}=100$. 
Example 3. Antisymmetric, constant force oscillator.

This example corresponds to

$$
\frac{\mathrm{d}^{2} x}{\mathrm{~d} t^{2}}+\operatorname{sgn}(x)=0, \quad f(x)=\operatorname{sgn}(x)
$$

with initial conditions given in Eq. (2). $\operatorname{sgn}(x)$ is +1 and -1 for $x>0$ and $x<0$, respectively. As $\left|B_{2}\right|<1$, Eq. (19) we can be written as follows $f\left(x_{2}(\tau)\right)=\operatorname{sgn}\left(x_{2}(\tau)\right)=\operatorname{sgn}\left(\frac{A\left(1+B_{2}\right) \cos \tau}{1+B_{2} \cos 2 \tau}\right)$

The Fourier series expansion for $\operatorname{sgn}(\cos \tau)$ is

$$
\operatorname{sgn}(\cos \tau)=\sum_{n=0}^{\infty} \frac{(-1)^{n} 4}{(2 n+1) \pi} \cos [(2 n+1) \tau]
$$

From Eqs. (13), (63) and (64) we obtain $b_{1}$ and $b_{3}$ as follows

$$
\begin{gathered}
b_{1}=\frac{4}{\pi} \\
b_{3}=-\frac{4}{3 \pi}
\end{gathered}
$$

Substituting Eqs. (17), (18), (65) and (66) into Eqs. (15) and (16), and solving the resulting equations for $B_{2}$ and $\omega$, yield

$$
\begin{gathered}
B_{2}=\frac{27}{365}=0.0739726 \\
\omega_{2}(A)=\sqrt{\frac{2}{\pi A}\left(1+\sqrt{\frac{1-B_{2}}{1+B_{2}}}\right)}=\sqrt{\frac{27}{7 \pi A}}=\frac{1.108046}{\sqrt{A}}
\end{gathered}
$$

As we can see, for $B_{2}=0$, the approximate frequency for the first-order harmonic balance approximation is obtained $\left(\omega_{1}=2 / \sqrt{\pi A}\right)$ [1]

Therefore, the second approximation to the periodic solution of the nonlinear oscillator is given by the following equation

$$
\frac{x_{2}(t)}{A}=\frac{392 \cos [\sqrt{27 /(7 \pi A)} t]}{365+27 \cos [2 \sqrt{27 /(7 \pi A)} t]}
$$

From Eqs. (11) and (12) the Fourier series expansion for the second-order approximate solution given in Eq. (69) is

$$
\frac{x_{2}(t)}{A}=\frac{28}{27} \cos \omega_{2} t-\frac{28}{729} \cos 3 \omega_{2} t+
$$

$$
\begin{aligned}
& +\frac{28}{19683} \cos 5 \omega_{2} t-\frac{28}{531441} \cos 7 \omega_{2} t+\ldots \\
& =1.03704 \cos \omega_{2} t-0.0384088 \cos 3 \omega_{2} t+ \\
& +0.0014226 \cos 5 \omega_{2} t-0.000052687 \cos 7 \omega_{2} t+\ldots
\end{aligned}
$$

which has an infinite number of harmonics.

For this nonlinear problem, the exact frequency and periodic solution are $[53,63]$

$$
\begin{gathered}
\omega_{e}(A)=\frac{\pi}{2 \sqrt{2 A}}=\frac{1.110721}{\sqrt{A}} \\
x_{e}(t)=\left\{\begin{array}{cc}
-\frac{t^{2}}{2}+A, & 0 \leq t \leq \frac{T_{e}}{4} \\
\frac{t^{2}}{2}-2 \sqrt{2 A} t+3 A, & \frac{T_{e}}{4}<t \leq \frac{3 T_{e}}{4} \\
-\frac{t^{2}}{2}+4 \sqrt{2 A} t-15 A, & \frac{3 T_{e}}{4}<t \leq T_{e}
\end{array}\right.
\end{gathered}
$$

An easy and direct calculation gives the following series representation for the exact solution $x_{e}(t)$

$$
x_{e}(t)=\frac{32 A}{\pi^{3}} \sum_{n=0}^{\infty} \frac{(-1)^{n}}{(2 n+1)^{3}} \cos \left[(2 n+1) \omega_{e} t\right]
$$

The first terms of the Fourier expansion in Eq. (73) are

$$
\begin{aligned}
\frac{x_{e}(t)}{A} & =1.03205 \cos \omega_{e} t-0.03822 \cos 3 \omega_{e} t \\
& +0.008256 \cos 5 \omega_{e} t-0.003009 \cos 7 \omega_{e} t+\ldots
\end{aligned}
$$

If we compare the first two terms of the Fourier series expansions of $x_{2}(t) / A$ (Eq. (70) and $x_{e}(t) / A$ (Eq. (74)) we can see that the relative errors for these terms are $0.48 \%$ and $0.49 \%$, respectively. The percentage error $(P E)$ of the approximate frequency in relation to the exact one is

$$
P E=\left|\left(\omega_{e}-\omega_{2}\right) / \omega_{e}\right| \cdot 100=0.24 \%
$$

The $0.24 \%$ accuracy is remarkably good.

$\mathrm{Wu}$ et al. [53] approximately solved Eq. (62) using an improved harmonic balance method (LHBM). They achieved the following results for the second approximation order

$$
\omega_{W S L 2}=\sqrt{\frac{104}{27 \pi A}}=\frac{1.107286}{\sqrt{A}}(P E=0.31 \%)
$$


Beléndez et al. [63] approximately solved Eq. (62) using He's homotopy perturbation method (HPM). They achieved the following results for the second approximation order

$\omega_{B 2}=\sqrt{\frac{2+2 \sqrt{4-\pi}}{\pi A}}=\frac{1.107452}{\sqrt{A}}$

$$
(P E=0.30 \%)
$$

The normalized periodic exact solution, $x_{e} / A$, achieved using Eq. (72) and the proposed second-order approximate solution, $x_{2} / A$ (Eq. (69)) are plotted in Figure 11. In Figure 12 we plotted the difference $\left(x_{e}-x_{2}\right) / A$.

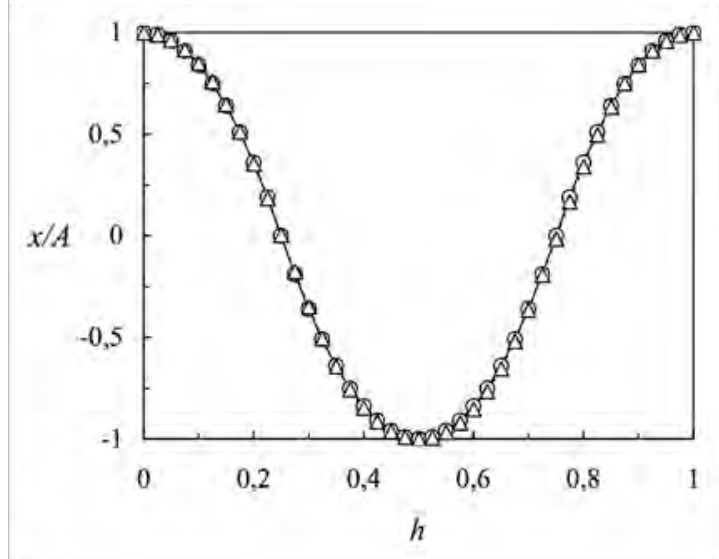

Fig. 11 Normalized approximate solution (triangles and dashed line) and exact solution (circles and continuous line) for the example 3.

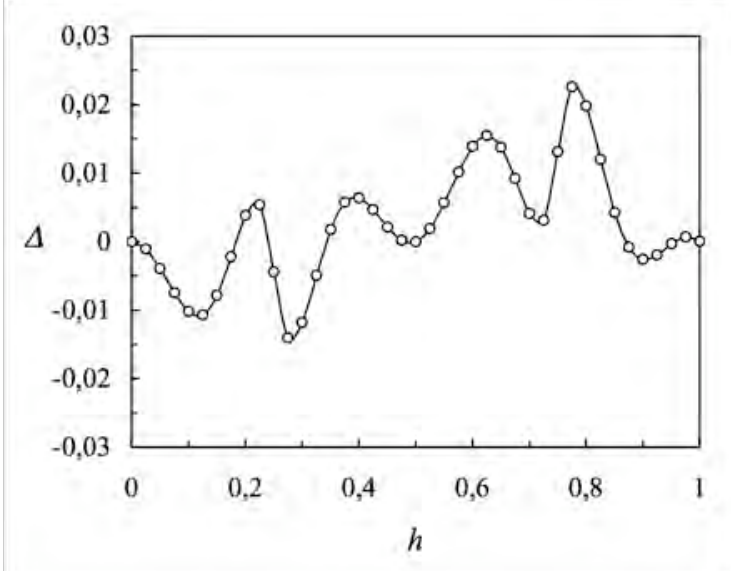

Fig. 12 Difference between normalized exact and second order approximate solutions (example 3).
Figures 11 and 12 show that Eqs. (68) and (69) provide excellent approximations to the exact periodic solution.

Example 4. Nonlinear oscillator in which the restoring force is inversely proportional to the dependent variable.

This oscillator is governed by the following second order nonlinear differential equation

$$
\frac{\mathrm{d}^{2} x}{\mathrm{~d} t^{2}}+\frac{1}{x}=0, \quad f(x)=\frac{1}{x}
$$

with initial conditions given in Eq. (2).

Eq. (19) we can be written as follows

$$
\begin{aligned}
f\left(x_{2}(\tau)\right) & =\frac{1}{x_{2}(\tau)}= \\
& =\frac{1-B_{2}}{A\left(1+B_{2}\right)} \frac{1}{\cos \tau}+\frac{2 B_{2}}{A\left(1+B_{2}\right)} \cos \tau \text { (78) }
\end{aligned}
$$

The Fourier series expansion for $\cos ^{-1} \tau$ is

$$
\cos ^{-1} \tau=\sum_{n=0}^{\infty}(-1)^{n} 2 \cos [(2 n+1) \tau]
$$

From Eqs. (13), (78) and (79) we obtain $b_{1}$ and $b_{3}$ as follows

$$
\begin{gathered}
b_{1}=\frac{2}{A\left(1+B_{2}\right)} \\
b_{3}=-\frac{2\left(1-B_{2}\right)}{A\left(1+B_{2}\right)}
\end{gathered}
$$

Substituting Eqs. (17), (18), (80) and (81) into Eqs. (15) and (16), and solving the resulting equations for $B_{2}$ and $\omega$, we obtain

$$
\begin{gathered}
B_{2}=\frac{1}{3}\left[2-\frac{296}{(81 \sqrt{4017}-649)^{1 / 3}}+\right. \\
\left.+(81 \sqrt{4017}-649)^{1 / 3}\right]=0.180593 \\
\omega_{2}(A)=\sqrt{\frac{\sqrt{1+B_{2}}+\sqrt{1-B_{2}}}{A^{2}\left(1+B_{2}\right)^{3 / 2}}}=\frac{1.246073}{A}
\end{gathered}
$$

As we can see, for $B_{2}=0$, the approximate frequency for the first-order harmonic balance approximation is obtained $\left(\omega_{1}=\sqrt{2} / A\right)[64,65]$.

Therefore, the second approximation to the periodic solution of the nonlinear oscillator is given by the following equation 


$$
\frac{x_{2}(t)}{A}=\frac{1.180593 \cos \left(1.246073 A^{-1} t\right)}{1+0.180593 \cos \left(2.492146 A^{-1} t\right)}
$$

From Eqs. (11) and (12) the Fourier series expansion for the second-order approximate solution given in Eq. (84) is

$$
\begin{aligned}
& \frac{x_{2}(t)}{A}=1.09104 \cos \omega_{2} t-0.0993342 \cos 3 \omega_{2} t+ \\
& +0.00904388 \cos 5 \omega_{2} t-0.0008234 \cos 7 \omega_{2} t+\ldots
\end{aligned}
$$

As we can see, Eq. (85) gives an expression that approximates all of the harmonics in the exact solution whereas the usual harmonic balancing techniques provide and approximation to only the lowest harmonic components.

For this problem the exact frequency is [64]

$$
\omega_{e}(A)=\frac{\sqrt{2 \pi}}{2 A}=\frac{1.253314}{A}
$$

and the percentage error $(P E)$ of the approximate frequency $\omega_{2}$ in relation to the exact one is

$$
P E=\left|\left(\omega_{e}-\omega_{2}\right) / \omega_{e}\right| \cdot 100=0.58 \%
$$

The $0.58 \%$ accuracy is remarkably good.

In order to approximately solve Eq. (77), Mickens rewrote this equation in a form that does not contain the $x^{-1}$ expression [64]

$$
x \frac{\mathrm{d}^{2} x}{\mathrm{~d} t^{2}}+1=0
$$

Using the second-order standard harmonic balance approximation to the periodic solution of Eq. (87), he obtained the second-order approximate frequency [64]

$\omega_{M 2}(A)=\frac{\sqrt{162}}{10 A}=\frac{1.272792}{A}(P E=1.6 \%)$

Beléndez et al [65] solved Eq. (77) applying the standard harmonic balance method. Using the second-order approximation, they obtained the approximate frequency

$$
\omega_{B 2}(A)=\frac{1.23733}{A} \quad(P E=1.3 \%)
$$

The (numerical) normalized exact periodic solution, $x_{e} / A$, obtained by numerically integrating Eq. (77) and the proposed secondorder approximate solution $x_{2} / A$ (Eq. (84)) are plotted in Figure 13 as a function of $h$.

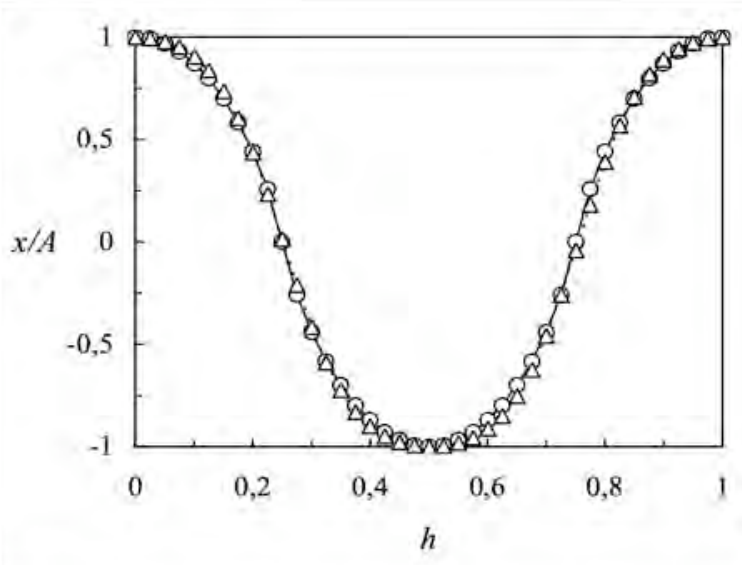

Fig. 13 Normalized approximate solution (triangles and dashed line) and exact solution (circles and continuous line) for the example 4.

In Figure 14 we plotted the difference $\left(x_{e}-x_{2}\right) / A$. These figures show that Eqs. (83) and (84) can provide high accurate approximations to the exact frequency and the exact periodic solution. These results are an indication of the accuracy of the proposed modified generalized harmonic balance method as applied to this particular problem and show that it provides an excellent approximation to the solution of Eq. (77).

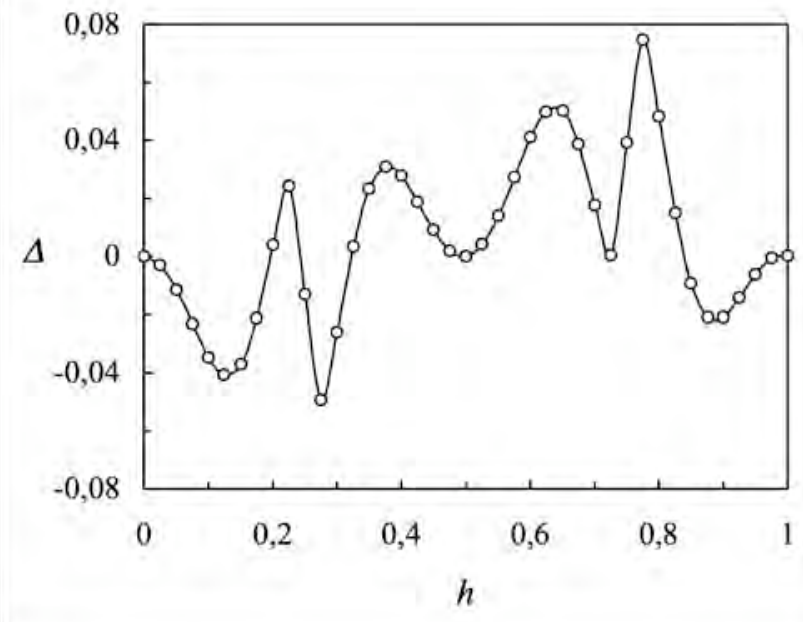

Fig. 14 Difference between normalized exact and approximate solutions (Example 4). 


\section{Conclusions}

Based on the rational harmonic balance method a new procedure has been developed and used to determine analytical approximate solutions of conservative nonlinear oscillators with odd nonlinearities. Unlike the classical harmonic balance techniques, in this new procedure the approximate solution and the restoring force are expanded in Fourier series prior to substituting them in the nonlinear differential equation. Four examples have been presented to illustrate excellent accuracy of the analytical approximate frequencies. The analytical representations obtained using this technique give excellent approximations to the exact solutions for the whole range of values of oscillation amplitudes. For a simple cubic oscillator, it has been shown that the relative error of the second-order analytical approximate frequency obtained using the approach considered in this paper is as low as $0.0089 \%$. In summary, this new procedure to apply the rational harmonic balance method is very simple in its principle, and it can be used to solve other nonlinear oscillators.

\section{References}

[1] R. E. Mickens, Oscillations in Planar Dynamics Systems (World Scientific, Singapore 1996).

[2] A. H. Nayfeh, Problems in Perturbations (Wiley, New York 1985).

[3] J. H. He, "Variational approach for nonlinear oscillators", Chaos, Solitons \& Fractals 34 (2007) 1430-1439.

[4] M. Dehghan and M. Tatari, "Te use of He's variational iteration method for solving multipoint boundary value problems", Phys. Scr. 72 (2007) 672-676.

[5] M. Rafei, D. D. Ganji, H. Daniali and H. Pashaei, "The variational iteration method for nonlinear oscillators with discontinuities", $J$. Sound. Vib. 305 (2007) 614-620.

[6] J. H. He, "Variational iteration method - a kind of non-linear analytical technique: some examples", Int. J. Non-linear Mech. 34 (1999) 699-708.

[7] J. I. Ramos, "On the variational iteration method and other iterative techniques for nonlinear differential equations", Appl. Math. Comput. 199 (2008) 39-69.

[8] J. H. He, X. H. Wu, "Construction of solitary solution and compact on-like solution by variational iteration method", Chaos, Solitons \& Fractals 29 (2006) 108-113.

[9] L. Xu, "Variational approach to the nonlinear oscillator of a mass attached to a stretched elastic wire", Phys. Scr. 77, 045006 (2008).

[10] V. Marinca, N. Herisanu and C. Bota, "Application of the variational iteration method to some nonlinear one-dimensional oscillators", Meccanica 43 (2008) 75-79.

[11] E. Yusufoglu, "Variational iteration method for construction of some compact and noncompact structures of Klein-Gordon equations", Int. J. Non-linear Sci. Numer. Simulation 8 (2007) 153-158.

[12] A. Sadighi and D.D. Ganji, "Solution of the generalized nonlinear Boussinesq equation using homotopy perturbation and variational iteration methods", Int. J. Non-linear Sci. Numer. Simulat. 8 (2007) 435-443.

[13] R. Mokhtari, "Variational iteration method for solving nonlinear differential-difference equations", Int. J. Non-linear Sci. Numer. Simulation 9 (2008) 19-23.

[14] M. A. Noor and S. T. Mohyud-Din, "Variational iteration method for solving higher-order nonlinear boundary value problems using He's polynomials", Int. J. Non-linear Sci. Numer. Simulation 9 (2008) 141-156.

[15] A. Ramiar, D. D. Ganji and Q. Esmaili, "Homotopy perturbation method and variational iteration method for orthogonal 2-D and axisymmetric impinging jet problems", Int. J. Non-linear Sci. Numer. Simulation 9 (2008) 115-130.

[16] T. Ozis and C. Koroglu, "A novel approach for solving the Fisher equation using Expfunction method", Phys. Lett. A 372, 38363840 (2008).

[17] A. Bekir and A. Boz, "Exact solutions for a class of nonlinear partial differential equations using exp-function method", Int. J. Non-linear Sci. Numer. Simulation 8 (2007) 505-512.

[18] S. D. Zhu, "Exp-function method for the Hybrid-Lattice system", Int. J. Non-linear Sci. Numer. Simulation 8 (2007) 461-464.

[19] S. D. Zhu, "Exp-function method for the discrete $\mathrm{mKdV}$ lattice", Int. J. Non-linear Sci. Numer. Simulation 8 (2007) 465-468. 
[20] J. H. He, "Homotopy perturbation method for solving boundary value problems", Phys. Lett. A 350 (2006) 87-88.

[21] T. Özis and A. Yildirim, “A comparative study of He's homotopy perturbation method for determining frequency-amplitude relation of a nonlinear oscillator with discontinuities", Int. J. Non-linear Sci. Numer. Simulation 8 (2007) 243-248.

[22] J. H. He, "Homotopy perturbation method for bifurcation on nonlinear problems", Int. J. Non-linear Sci. Numer. Simulation 6 (2005) 207-208.

[23] A. Beléndez, A. Hernández, T. Beléndez, E. Fernández, M. L. Álvarez and C. Neipp, "Applica-tion of He's homotopy perturbation method to the Duffing-harmonic oscillator", Int. J. Non-linear Sci. Numer. Simulation 8 (2007) 79-88.

[24] A. Beléndez, A. Hernández, T. Beléndez, C. Neipp and A. Márquez, "Application of the homotopy perturbation method to the nonlinear pendulum, Eur. J. Phys. 28 (2007) 93-104.

[25] M. Gorji, D. D. Ganji and S. Soleimani, "New application of He's homotopy perturbation method", Int. J. Nonlin. Sci. Num.. 8 (2007), 319-328.

[26] F. Shakeri and M. Dehghan, "Inverse problem of diffusion by He's homotopy perturbation method", Phys. Scr. 75 (2007) 551-556.

[27] A. Beléndez, A. Hernández, T. Beléndez, A. Márquez and C. Neipp, "Application of He's homotopy perturbation method to conservative truly nonlinear oscillators", Chaos Solitons \& Fractals 37 (2008), 770-780.

[28] M. M. Mousa and S. F. Ragab, Application of the homotopy perturbation method to linear and nonlinear Schrödinger equation, $Z$. Naturforsch. 63a (2008), 140-144.

[29] M. T. Darvishi and F. Khani, Application of He's homotopy perturbation method to stiff systems of ordinary differential equations, $Z$. Naturforsch. 63a, (2008), 19-23.

[30] E. Yusufoglu, "Homotopy perturbation method for solving a nonlinear system of second order boundary value problems", Int. J. Non-linear Sci. Numer. Simulation 9 (2008) 353-358.

[31] A. Beléndez, C. Pascual, D. I. Méndez and A. Márquez, “Application of He's homotopy perturbation method to the relativistic (an)harmonic oscillator. I: Comparison between approximate and exact frequencies", Int. J. Nonlin. Sci. Num.8 (2007) 483-491.

[32] A. Beléndez, C. Pascual, D. I. Méndez, M. L. Alvarez and C. Neipp, "Application of He's homotopy perturbation method to the relativistic (an)harmonic oscillator. II: A more accurate approximate solution", Int. J. Nonlinear Sci. Numer. Simulation 8 (2007), 493504.

[33] M. Mahmood, M. A. Hossain, S. Asghar et al., "Application of homotopy perturbation method to deformable channel with wall suction and injection in a porous medium", Int. J. Non-linear Sci. Numer. Simulation 9 (2008) 195-206.

[34] A. Yildirim, "Exact solutions of nonlinear differential-difference equations by He's homotopy perturbation method", Int. J. Nonlinear Sci. Numer. Simulation 9 (2008) 111114.

[35] A. Beléndez, E. Gimeno, E. Fernández, D. I. Méndez and M. L. Alvarez, "Nonlinear oscillator in which the restoring force is inversely proportional to the dependent variable", Phys. Scr. 77 (2008) 065004.

[36] A. Beléndez, A. Hernández, T. Beléndez, A. Márquez and C. Neipp, "An improved 'heuristic' approximation for the period of a nonlinear pendulum: linear analysis of a nonlinear problem", Int. J. Non-linear Sci. Numer. Simulation 8 (2007) 329-334.

[37] A. Beléndez, C. Pascual, C. Neipp, T. Beléndez and A. Hernández, "An equivalent linearization method for conservative nonlinear oscillators", Int. J. Non-linear Sci. Numer. Simulation 9 (2008) 9-17.

[38] J. H. He, "A new perturbation technique which is also valid for large parameters", $J$. Sound Vib. 229 (2000) 1257-1263.

[39] T. Özis and A. Yildirim, "Determination of periodic solution for a $u^{1 / 3}$ force by He's modified Lindstedt-Poincaré method"; $J$. Sound Vib. 301 (2007) 415-419.

[40] J. H. He, "Modified Lindstedt-Poincare methods for some non-linear oscillations. Part I: expansion of a constant", Int. J. Non-linear Mech. 37 (2002) 309-314.

[41] J. H. He, "Modified Lindstedt-Poincare methods for some non-linear oscillations. Part III: double series expansion", Int. J. Nonlinear Sci. Numer. Simulation 2 (2001) $317-$ 320 . 
[42] H. M. Liu, “Approximate period of nonlinear oscillators with discontinuities by modified Lindstedt-Poincaré method", Chaos, Solitons \& Fractals 23 (2005) 577-579.

[43] J. H. He, "Some asymptotic methods for strongly nonlinear equations", Int. J. Mod. Phys. B 20(2006), 1141-1199.

[44] J. I. Ramos, “On Lindstedt-Poincaré techniques for the quintic Duffing equation", Appl. Math. Comput. 193 (2007), 303-310.

[45] J. I. Ramos, An artificial parameterdecomposition method for nonlinear oscillators: Applications to oscillators with odd nonlinearities, J. Sound Vib. 307 (2007), 312329.

[46] J. H. He, "A modified perturbation technique depending upon an artificial parameter", Meccanica 35 (2000) 299-311.

[47] J. H. He, "Application of parameterexpanding method to strongly nonlinear oscillators", Int. J. Non-linear Sci. Numer. Simulation 8 (2007), 121-124.

[48] S. Q. Wang and J. H. He, "Nonlinear oscillator with discontinuity by parameterexpansion method", Chaos, Solitons \& Fractals 35 (2008) 688-691.

[49] L. Xu, "Application of He's parameterexpansion method to an oscillation of a mass attached to a stretched elastic wire", Phys. Lett. A 368, 259-262 (2007).

[50] D. H. Shou, J. H. He, "Application of parameter-expanding method to strongly nonlinear oscillators", Int. J. Non-linear Sci. Numer. Simulation 8 (2007) 121-124.

[51] C.W. Lim, B.S. Wu, W.P. Sun, "Higher accuracy analytical approximations to the Duffing-harmonic oscillator", J. Sound Vib. 296 (2006) 1039-1045.

[52] A. Beléndez, A. Hernández, A. Márquez, T. Beléndez and C. Neipp, "Analytical approximations for the period of a simple pendulum", Eur. J. Phys. 27 (2006) 539-551.

[53] B. S. Wu, W. P. Sun and C. W. Lim, "An analytical approximate technique for a class of strongly non-linear oscillators", Int. J. Nonlinear Mech. 41, 766-774 (2006).

[54] A. Beléndez, C. Pascual, D.I. Méndez and C. Neipp, "Solution of the relativistic (an)harmonic oscillator using the harmonic balance method", J. Sound Vib. 311 (2008) 1447-1456.

[55] A. Beléndez, C. Pascual, "Harmonic balance approach to the periodic solutions of the (an)harmonic relativistic oscillator", Phys. Lett. A 371 (2007) 291-299.

[56] B.S. Wu, P.S. Li, "A method for obtaining approximate analytic periods for a class of nonlinear oscillators", Meccanica 36 (2001) 167-176.

[57] J. H. He, Non-perturbative methods for strongly nonlinear problems (dissertation.deVerlag im Internet GmbH, Berlin 2006).

[58] J. H. He, "An elementary introduction to recently developed asymptotic methods and nanomechanics in textile engineering", Int. J. Mod. Phys. B 22 (2008)3487-3578.

[59] K. Cooper and R. E. Mickens, "Generalized harmonic balance/numerical method for determining analytical approximations to the periodic solutions of the $x^{4 / 3}$ potential", $J$. Sound. Vib. 250 (2002) 951-954.

[60] R. E. Mickens, "A generalization method of harmonic-balance", J. Sound Vib. 111, 515518 (1986).

[61] R. E. Mickens and D. Semwogerere, "Fourier analysis of a rational harmonic balance approximation for periodic solutions", $J$. Sound Vib. 195, 528-530 (1996).

[62] A. Beléndez, C. Pascual, E. Fernández, C. Neipp and T. Beléndez, "Higher-order approximate solutions to the relativistic and Duffing-harmonic oscillators by modified He's homotopy methods", Phys. Scr. 77 (2008) 025004.

[63] A. Beléndez, A. Hernández, T. Beléndez, C. Neipp and A. Márquez, "Higher accuracy analytical approximations to a nonlinear oscillator with discontinuity by He's homotopy perturbation method", Phys. Lett. A 372, 20102016 (2008).

[64] R. E. Mickens, "Harmonic balance and iteration calculations of periodic solutions to $y^{\prime \prime}+y^{-1}=0$ ", J. Sound Vib. 306, 968-972 (2007).

[65] A. Beléndez, D. I. Méndez, T. Beléndez, A. Hernández and M. L. Alvarez, "Harmonic balance approaches to the nonlinear oscillators in which the restoring force is inversely proportional to the dependent variable", $J$. Sound Vib. 314, 775-782 (2008). 